\title{
Viscous surface flow induced on Ti-based bulk metallic glass by heavy ion irradiation
}

\author{
Kun Zhang ${ }^{\mathrm{a}}$, Zheng $\mathrm{Hu}^{\mathrm{a}}{ }^{\mathrm{a} b}$, Fengjiang $\mathrm{Li}^{\mathrm{a}}$, Bingchen Wei ${ }^{\mathrm{a}, *}$ \\ ${ }^{a}$ Key Laboratory of Microgravity (National Microgravity Laboratory), Institute of Mechanics, Chinese Academy of Sciences, Beijing 100190, China \\ b Science and Technology on Vehicle Transmission Laboratory, China North Vehicle Research Institute, Beijing 100072, China
}

\section{A R T I C L E I N F O}

\section{Article history:}

Received 11 May 2016

Accepted 18 August 2016

Available online 21 August 2016

\section{Keywords:}

Metallic glass

Ion irradiation

Viscous flow

\begin{abstract}
A B S T R A C T
Ti-based bulk metallic glass was irradiated by a $20 \mathrm{MeV} \mathrm{Cl}^{4+}$ ion beam under liquid-nitrogen cooling, which produced remarkable surface smoothing and roughening that respectively correspond to normal and off-normal incidence angles of irradiation. Atomic force microscopy confirms two types of periodic ripples distributed evenly over the rough glass surface. In terms of mechanism, irradiation-induced viscosity agrees with the theoretical prediction for metallic glasses near glass transition temperature. Here, a model is introduced, based on relaxation of confined viscous flow with a thin liquid-like layer, that explains both surface smoothing and ripple formation. This study demonstrates that bulk metallic glass has high morphological instability and low viscosity under ion irradiation, which assets can pave new paths for metallic glass applications.
\end{abstract}

(c) 2016 Published by Elsevier B.V.

\section{Introduction}

Because of their unique mechanical and chemical properties, such as high strength and superior resistance to corrosion, metallic glasses (MGs) have attracted great interest in recent decades [1-5]. Due to their inherent disordered structure, they are supposed to be ideal potential candidates for application in nuclear-irradiated environments or deep space. Many studies focus on intrinsic irradiation responses by MGs, including damage morphology [6], enhanced mechanical performance [7] and local structural change $[8,9]$. Studies show that the ion beam resistance capability of MGs is much improved over corresponding crystalline counterparts and even traditional radiation resistance materials [10,11]. As an effective means for surface smoothing and pattern formation, ion beam irradiation has been widely used on amorphous materials, such as $\alpha-\mathrm{Si}, \alpha-\mathrm{SiO}_{2}$ and $\alpha-\mathrm{C}$ films [12-14]. Most theories [15-17] describing the evolution of surface morphologies involve a competition between smoothing mechanisms, such as surface diffusion or viscous flow, and roughening mechanisms, such as sputtering or deposition. The named processes are of importance for various aspects of thin amorphous film science and micro-fabrication $[18,19]$. However, research is lacking on surface smoothing and MG morphology evolution. Mayr and Shao found that ion irradiation of MG films, in appropriate doses, can remove superficial mountain-

\footnotetext{
* Corresponding author.

E-mail address: weibc@imech.ac.cn (B. Wei).
}

like features and reduce the mean surface roughness [20,21]. Mayr proposed that viscous flow is the dominant surface relaxation mechanism during surface smoothing [22,23]. Thomas demonstrated that surface roughness of Fe-Ni films initially decreases, then increases at increased fluence levels. They attributed change in surface morphology to interplay between ion-induced sputtering, volume diffusion and surface diffusion [24]. To explain the ultra-smoothness of MG films, other mechanisms have also been proposed, such as ballistic effect [14,25]. Actually, surface morphology evolution of irradiated MGs is rather complex, as it depends on incident angle, ion energy and target material. Regarding formation of patterns and ripples during heavy ion irradiation, the kinetics of MG surface smoothing is still unclear. Thus far, most irritation experiments were carried out on thin MG samples just several or dozens of micrometers in scale, which thin films may be not stable when constrained on a substrate, causing the intrinsic irradiated response of the material itself to be hidden or attenuated [26,27].

In this work, the surface morphology of $\mathrm{Ti}_{40} \mathrm{Zr}_{25} \mathrm{Be}_{30} \mathrm{Cr}_{5}$ bulk MGs under $\mathrm{Cl}^{4+}$ ion irradiation $(20 \mathrm{MeV})$ is studied. Surface smoothing and ripple formation occur corresponding respectively to normal and off-normal incidence. The kinetics and related mechanism for smoothing and ripple formation are discussed.

\section{Experiments}

Ti-based bulk MGs with $5 \mathrm{~mm}$ diameter were prepared by arc melting a mixture of elements with purity higher than $99.9 \%$ in an argon atmosphere and then chilled casting into a copper mold. 


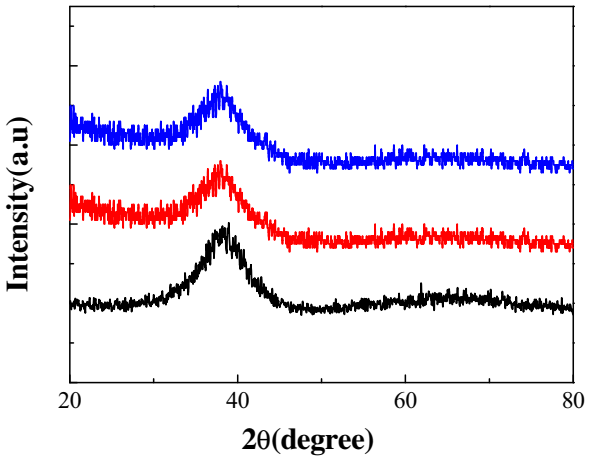

Fig. 1. XRD diagrams of Ti-based MGs (black- as-cast, red- normal irradiation, blue, off-normal irradiation). (For interpretation of the references to colour in this figure legend, the reader is referred to the web version of this article.)

Specimens $5 \mathrm{~mm}$ in length were cut from the resulting rods. Prior to irradiation experiments, surfaces of the samples were mechanically polished to a mirror finish and cleaned ultrasonically. Next, the prepared samples were irradiated at two incident angles $\left(0^{\circ}\right.$ normal incidence, $70^{\circ}$ off-normal incidence) at a fluence of $1.6 \times 10^{16}$ ions $/ \mathrm{cm}^{2}$, while parameters of the ion beams and displacement damage were calculated using SRIM2008 code [19]. During irradiation, beam size was about $2.5 \times 1.8 \mathrm{~cm}^{2}$ and the sample holder was cooled by liquid nitrogen. The X-ray diffraction (XRD) analysis was carried out on a Philips PW 1050 diffractometer using $\mathrm{CuK} \alpha$ radiation, whereas morphology was tested by scanning electron microscope (SEM, JEM-2100F) and atomic force microscope (AFM, Hysitron Tribo Scope).

\section{Results and discussions}

Shown in Fig. 1 are XRD patterns of the as-cast Ti-based MG, as well as MGs irradiated at normal and off-normal incidences. In said figure, both the irradiated and untreated Ti-based MGs clearly exhibit only one broad diffraction peak in the XRD curves, confirming amorphous structure at near-surface. However, the peak intensity of the irradiated sample exhibits a slight drop when compared with the control sample, demonstrating that the amorphous state becomes more disordered as the accelerated $\mathrm{Cl}^{4+}$ ion sets a large number of matrix atoms into motion.

In Fig. 2 appear SEM images of as-cast and irradiated and untreated Ti-based MG samples at a fluence of $1.6 \times 10^{16}$ ions $/ \mathrm{cm}^{2}$, wherein the untreated sample is essentially featureless, having a relatively homogeneous surface without obvious intrinsic defects. By contrast, obvious surface features appear on the Ti-based specimen surface after normal irradiation [28]. As shown in Fig. 2b, there are two main kinds of damage pits on the irradiated surface: one crater-like with an average diameter less than $3 \mathrm{um}$, and the other, blister-like, possessing a much larger size ranging from 5 to $20 \mathrm{um}$.
The size of the crater-like damage pits is different from others produced by energetic ion and cluster ion impact in other metallic materials $[29,30]$. The "damaged" region should be viewed as an intrinsic defect of most MGs, which is caused by the interaction of the high-energy ion beam and amorphous states. Interestingly, both the "damaged" and "matrix" regions have relatively smooth surfaces, which is not true in the case of the off-normal system. As shown in Fig. 2c, two kinds of damage pits also appear on the irradiated surface and the surface of the irradiated region is uneven. A periodic ripple or wavelike pattern appears in both the blister-like and matrix regions, with a spatial periodicity over the off-normal ion-bombarded surface. It should be noted that the surface ripples may flow along the ion beam in the "crater-like features" region, concealing the "damaged" characteristic.

To further demonstrate the morphology of surface smoothing and roughening, AFM was introduced to the irradiated systems. As shown in Fig. 3, the surface is relatively smooth in both the "matrix" and "damaged" regions after normal irradiation, with mean surface roughness values of $12.3,6.6,5.1,3.4$ and $3.6 \mathrm{~nm}$ over increasing irradiation time. It is evident that the mean surface roughness of Tibased MG decreases with increasing dose within the tested fluence range, which confirms smoothing of surfaces.

Fig. 4a and b details AFM images of surface features produced by off-normal irradiation. Interestingly, two kinds of ripples appear, while the propagation direction of the wave vector is parallel to the incidence of the ion beam. The surface ripples in the matrix region have a broad wavelength $(1300 \mathrm{~nm})$ and amplitude $(350 \mathrm{~nm})$, while the ripple in the damaged region is compressed, with a wavelength of about $467 \mathrm{~nm}$ and an amplitude of $30 \mathrm{~nm}$. Fig. 4c shows the adjoining region between the damaged and matrix states, in which a valley formed with maximum depth of $2.5 \mathrm{um}$ at the adjoining position, close to the penetration depth of the $\mathrm{Cl}^{4+}$ ion $(1.7 \mathrm{um}$, calculated by SRIM2008 code).

Notably, the process of surface smoothing and roughing in solids is rather complex, depending as it does on the properties of the incident ion, incident angle and target solid. For low-energy ion irradiation, the projectiles are implanted the near surface. In this case, the main cause of surface roughening is believed to be surface erosion or deposition, while surface smoothing appears to be due to surface diffusion or viscous flow. In our studies $(E=20 \mathrm{MeV})$, the calculated number of sputtered atoms per incident ion under highenergy heavy ion irradiation is less than 1 for heavy ions [31,32]. Moreover, surface smoothing and roughening appear at a relatively low temperature, indicating that macroscopic thermal effect and diffusion are unimportant.

Many recent studies indicate that amorphous materials behave in a viscoelastic manner during heavy ion irradiation. Surface viscous flow may play a dominant quantitative role in surface diffusion and sputtering, which processes are driven by a Laplace stress that generates a particle current and causes viscosity to decrease its by action on the local surface. The radiation-induced viscosity, $\eta$, is extracted using the method developed by Snoeks et al. [33].

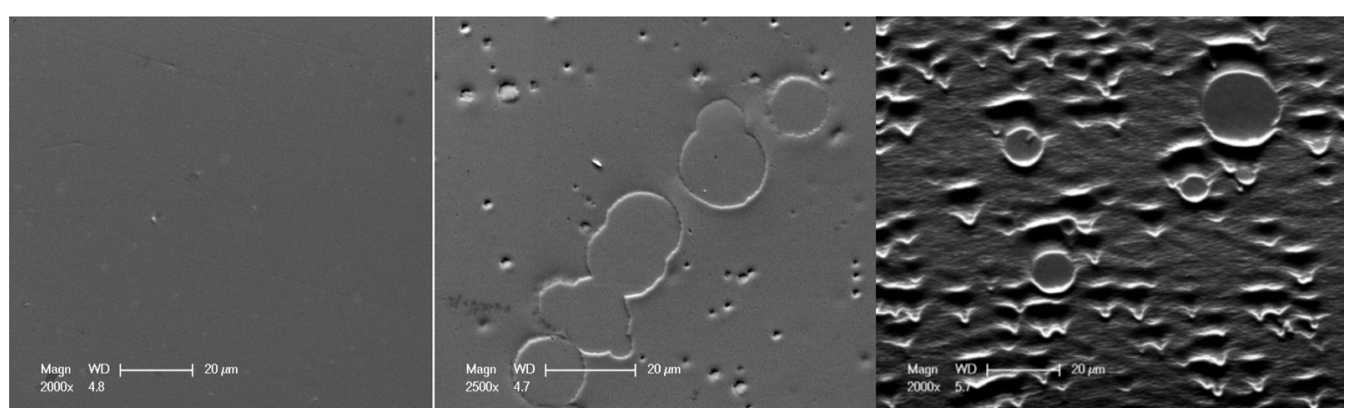

Fig. 2. Surface morphology of Ti-based MGs (a- as-cast, b- normal irradiation, c- off-normal irradiation). 

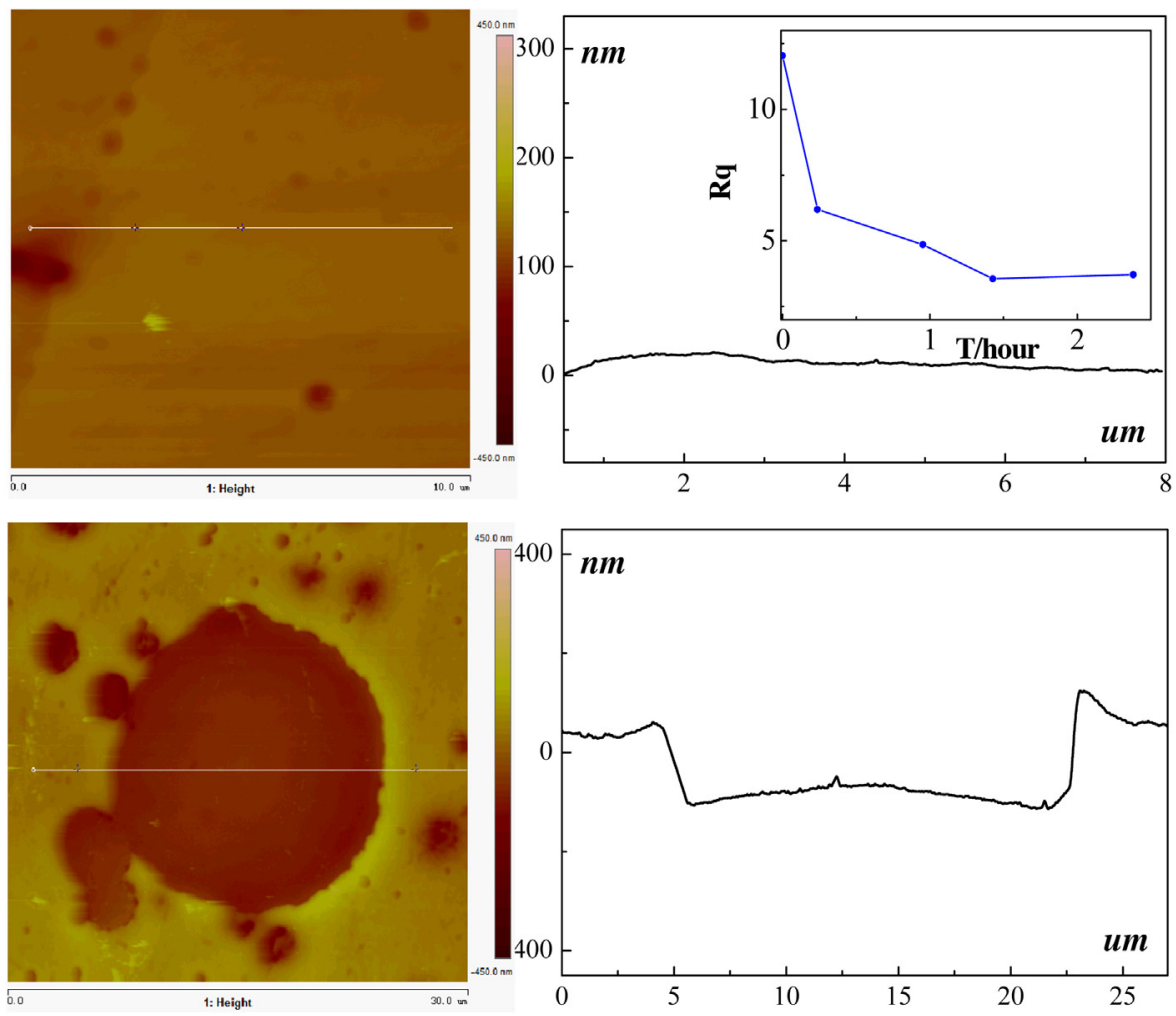

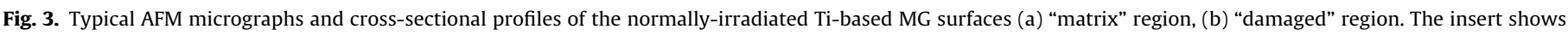
the evolution of surface roughness under increased displacements per atom (DPA).

Table 1

Experimental parameters of Ti-based MGs with irradiation under the model described by Eq. (1).

\begin{tabular}{llll}
\hline Sn $(\mathrm{keV} / \mathrm{um})$ & $\mathrm{k}(\mathrm{dpa} / \mathrm{s})$ & $v$ & $\mathrm{E}(\mathrm{GPa})$ \\
\hline 18.8 & $1.4 \times 10^{-3}$ & 0.35 & $120[24]$ \\
$\theta \mathrm{C}\left(10^{-6} \mathrm{~J} \mathrm{Km}^{-3}\right)$ & $\mathrm{r}(\mathrm{nm})$ & $\mathrm{D}\left(\mathrm{cm}^{2} / \mathrm{s}\right)$ & $\mathrm{t}(\mathrm{s})$ \\
3.0 & 5 & $1 \times 10^{-3}$ & $2 \times 10^{-11}$ \\
\hline
\end{tabular}

Radiation-induced viscosity in the amorphous solid is described by [34,35]:

$\eta=\frac{\Phi}{k} \frac{(7-5 v)}{10\left(1-v^{2}\right)} \frac{E \theta C \Delta T}{S_{n} e}$

where particle flux is represented by $\Phi$, displacement rates by $k$, Poisson ratio by $v$, elastic modulus by $E$, mass density by $\theta$, specific heat by $C$ and nuclear stopping power by $S_{n}$, which is derived from Monte Carlo calculations (TRIM2008). $\Delta T$ is a parameter that describes the increase of temperature within the cascade, and which is necessary to allow sufficient flow for plastic deformation. The resulting $\Delta T$ values can be obtained by the Eq. (2):

$$
\Delta \mathrm{T}=\frac{Q}{(4 \pi)^{1.5} \theta C} \frac{1}{(D t)^{1.5}} \exp \left(-\frac{r^{2}}{4 D t}\right)
$$

The relevant parameters are listed in Table 1. A flux of $1.6 \times 10^{16}$ ions $/ \mathrm{cm}^{2}$, corresponds to a viscosity of $\eta=4 \times 10^{12}$ Pa s, which is similar to the results found in MGs close to glass transition temperature [36]. Compared to the case for thermally-activated viscous flow, radiation-induced viscosity is significantly lower, which accounts for surface smoothing or roughening of Ti-based bulk MG systems.

For a detailed study of the relationship between the viscous flow and the surface relaxation mechanism, the evolution of power spectral density (PSD) was analyzed, which is easily accessible via measurements by different light scattering techniques or from Fourier analysis of height profiles measured by different imaging techniques. To some extent, the dominant relaxation process can be identified by the asymptotic wavenumber scaling of PSD. Fig. 5 summarizes the typical evolution of PSD during ion beam interaction. Starting from the initial surface PSD $(\mathrm{f}=0)$, an initial rapid decrease of the surface features wavenumber is observed, followed by a steady state, in which PSD grows according to a power law thus: $\operatorname{PSD}(\mathrm{f}=\mathrm{q} / 2 \pi) \propto \mathrm{q}^{-4}$. According to Orchard [37], in the applied equation, the $\mathrm{q}^{-4}$ dependence of PSD shows smoothing via viscous flow confined to the surface or via the mechanism of superficial diffusion [38]. In the case of smoothing by mechanism of confined viscous flow, the wavelength should increase with increasing ion energy, which prediction accords with our experimental data. The $\mathrm{q}^{-4}$ dependence appears under both normal and off-normal incidences, indicating that surface-confined viscous flow plays dominant role during $\mathrm{Cl}^{4+}$ ion irradiation of Ti-based bulk MGs, which result differs from those for thin MG film systems $[18,22]$. We speculate that surfaces of bulk MGs have a high morphological instability and low viscosity under $\mathrm{Cl}^{4+}$ ion irradiation. On one hand, the low viscosity of Ti-based MG results in surface smoothing by a vertical Laplace stress under normal incidence angle irradiation. On the other hand, the shear component of the Laplace stress induced by off-normal incidence gives rise to the observed surface roughening and formation of ripples.

\section{Conclusion}

Ti-based metallic glass was irradiated by $\mathrm{Cl}^{4+}$ ions under liquidnitrogen cooling. Obvious smoothing and roughening phases on the MG surface resulted, which correspond respectively to the normal and off-normal incidence angles. Atomic force microscopy confirms two types of periodic ripples distributed evenly over the rough surface. The irradiation-induced viscosity of MG is about $4 \times 10^{12} \mathrm{~Pa}$, 

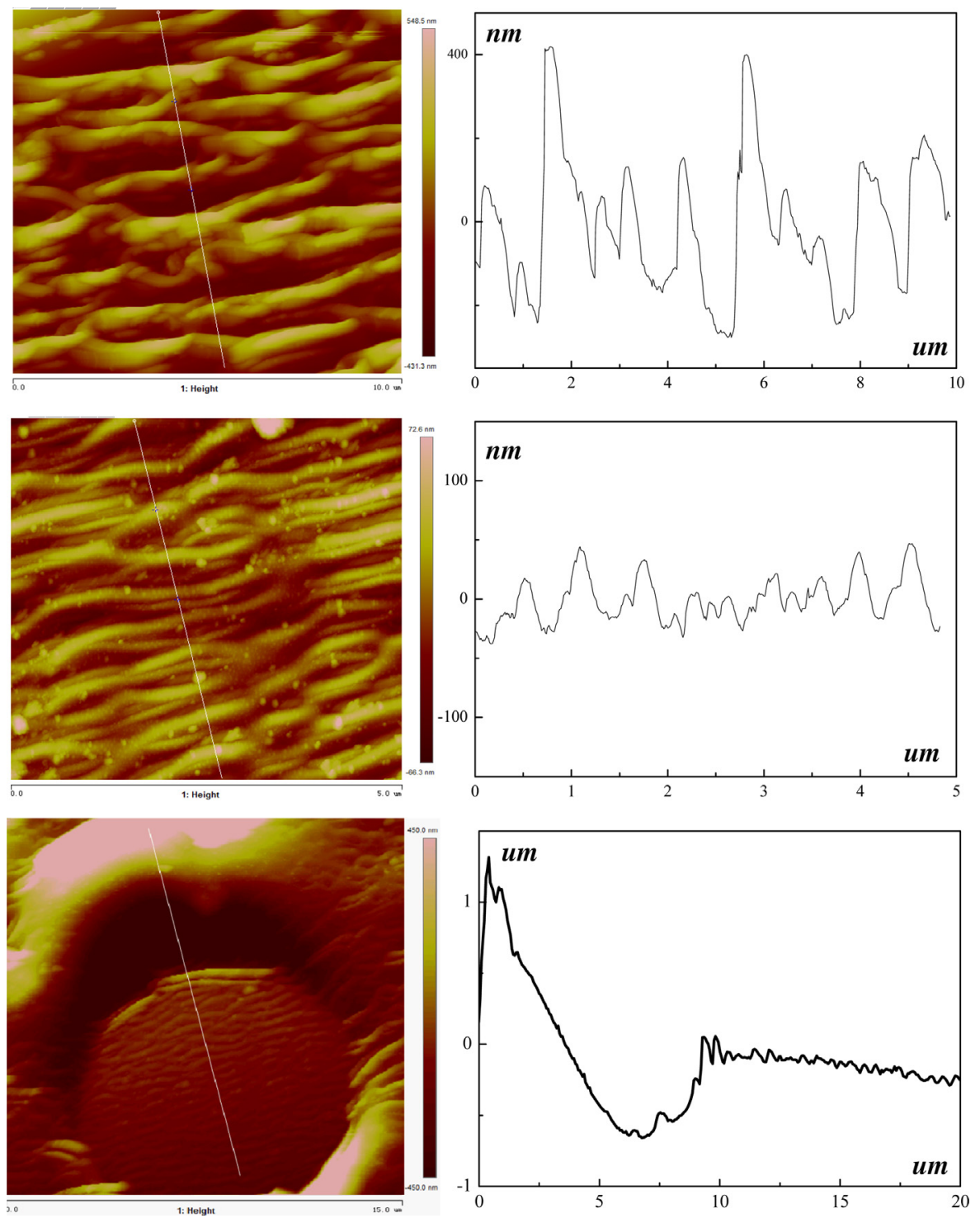

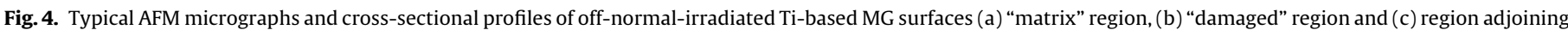
the "damaged" and "matrix" states. The solid line in Fig. 4 is the incidence direction of the ion beam.

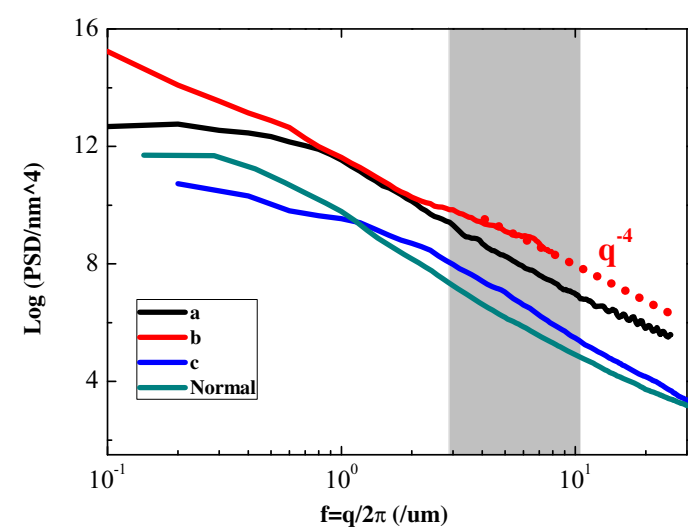

Fig. 5. PSD of ion-irradiated Ti-based MG surface at an ion dose $1.6 \times 10^{16} \mathrm{ions} / \mathrm{cm}^{2}$, calculated from respective AFM micrographs using nanoscope software: (a) "matrix" region, (b) "damaged" region, (c) region adjoining the "damaged" and "matrix" states and (d) normal incident region. The wavelength range is marked by a gray frame. which accords with the theoretical prediction for metallic glasses close to glass transition temperature. Surface-confined viscous flow plays a dominant quantitative role, which is due to radiationinduced softening of the low-viscosity surface layer.

\section{Acknowledgement}

The authors would like to acknowledge the support by the National Natural Science Foundation of China (Grant No. 51271193, No. 11332011 and No. 51401028).

\section{References}

[1] A. Greer, E. Ma, Bulk metallic glasses: at the cutting edge of metals research, MRS Bull. 32 (2007) 611-619.

[2] W.L. Johnson, M.D. Demetriou, J.S. Harmon, M.L. Lind, K. Samwer, Rheology and ultrasonic properties of metallic glass-forming liquids: a potential energy landscape perspective, MRS Bull. 32 (2007) 644-650.

[3] W.H. Wang, R.J. Wang, F.Y. Li, D.Q. Zhao, M.X. Pan, Elastic constants and their pressure dependence of $\mathrm{Zr}_{41} \mathrm{Ti}_{14} \mathrm{Cu}_{12.5} \mathrm{Ni}_{9} \mathrm{Be}_{22.5} \mathrm{C}_{1}$ bulk metallic glass, Appl. Phys. Lett. 74 (1999) 1803-1805.

[4] S.V. Ketov, Y.H. Sun, S. Nachum, et al., Rejuvenation of metallic glasses by non-affine thermal strain, Nature 524 (2015) 200-203. 
[5] J. Schroers, Processing of bulk metallic glass, Adv. Mater. 22 (2010) 1566-1597.

[6] M.D. Rodriguez, B. Afra, C. Trautmann, et al., Morphology of swift heavy ion tracks in metallic glasses, J. Non-Cryst. Solids 358 (3) (2012) 571-576.

[7] R. Raghavan, K. Boopathy, R. Ghisleni, et al., Ion irradiation enhances the mechanical performance of metallic glasses, Scr. Mater. 62 (2010) 462-465.

[8] C. Dimitrov, H. Dammak, P. Legrand, et al., Displacement damage and chemical disordering in short-range ordered alloys irradiated by swift heavy ions, Radiat. Effects Defects Solids 126 (1993) 115-118.

[9] M.T. Myers, S. Charnvanichborikarn, C.C. Wei, et al., Phase transition, segregation and nanopore formation in high-energy heavy-ion-irradiated metallic glass, Scr. Mater. 67 (2012) 887-890.

[10] W. Hou, X. Mei, Z. Wang, et al., Resistance to $\mathrm{He}^{2+}$ irradiation damage in metallic glass $\mathrm{Fe}_{80} \mathrm{Si}_{7.43} \mathrm{~B}_{12.57}$, Nucl. Instrum. Methods Phys. Res. Sect. B 342 (2015) 221-227.

[11] W.D. Luo, B. Yang, G.L. Chen, Effect of Ar+ ion irradiation on the microstructure and properties of $\mathrm{Zr}-\mathrm{Cu}-\mathrm{Fe}-\mathrm{Al}$ bulk metallic glass, Scr. Mater. 64 (2011) 625-628.

[12] S.A. Norris, Stability analysis of a viscoelastic model for ion-irradiated silicon, Phys. Rev. B 85 (2012) 155325.

[13] S.K. Srivastava, K. Ganesan, P. Gangopadhyay, et al., High energy ion irradiation induced surface patterning on a $\mathrm{SiO}_{2}$ glass substrate, Nucl. Instrum. Methods Phys. Res. Sect. B 338 (2014) 89-94.

[14] M. Moseler, P. Gumbsch, C. Casiraghi, et al., The ultrasmoothness of diamond-like carbon surfaces, Science 309 (2005) 1545-1548.

[15] T.M. Mayer, E. Chason, A.J. Howard, Roughening instability and ion-induced viscous relaxation of $\mathrm{SiO}_{2}$ surfaces, J. Appl. Phys. 76 (1994) 1633-1643.

[16] M.A. Makeev, R. Cuerno, A.L. Barabasi, Morphology of ion-sputtered surfaces, Nucl. Instrum. Methods Phys. Res. Sect. B 197 (2002) 185-227.

[17] W.L. Chan, E. Chason, Making waves: kinetic processes controlling surface evolution during low energy ion sputtering, J. Appl. Phys. 101 (2007) 121301

[18] S.G. Mayr, R.S. Averback, Surface smoothing of rough amorphous films by irradiation-induced viscous flow, Phys. Rev. Lett. 87 (2001) 196106.8.

[19] C.G. Zimmermann, M. Yeadon, K. Nordlund, et al., Burrowing of Co nanoparticles on clean Cu and Ag surfaces, Phys. Rev. Lett. 83 (1999) 1163.

[20] S.G. Mayr, R.S. Averback, Surface smoothing of rough amorphous films by irradiation-induced viscous flow, Phys. Rev. Lett. 87 (2001) 196106.

[21] L. Shao, D. Chen, M. Myers, et al., Smoothing metallic glasses without introducing crystallization by gas cluster ion beam, Appl. Phys. Lett. 102 (2013) 101604

[22] S.G. Mayr, Y. Ashkenazy, K. Albe, et al., Mechanisms of radiation-induced viscous flow: role of point defects, Phys. Rev. Lett. 90 (2003) 055505.
[23] S.G. Mayr, Y. Ashkenazy, R.S. Averback, Evolution of thin-film morphologies in metals during ion beam bombardment, Nucl. Instrum. Methods Phys. Res. Sect. B 212 (2003) 246-252.

[24] H. Thomas, S. Thomas, R.V. Ramanujan, et al., Swift heavy ion induced surface and microstructural evolution in metallic glass thin films, Nucl. Instrum. Methods Phys. Res. Sect. B 287 (2012) 85-90.

[25] S. Vauth, S.G. Mayr, Relevance of surface viscous flow, surface diffusion, and ballistic effects in keV ion smoothing of amorphous surfaces, Phys. Rev. B 75 (2007) 224107

[26] A. Gutzmann, S. Klaumünzer, A. Benyagoub, et al., Influence of a mechanical stress on the plastic deformation of glassy $\mathrm{Fe}_{40} \mathrm{Ni}_{40} \mathrm{~B}_{20}$ during heavy ion irradiation, Radiat. Effects Defects Solids 126 (1993) 133-136.

[27] A. Gutzmann, S. Klaumünzer, P. Meier, Ion-beam-induced surface instability of glassy $\mathrm{Fe}_{40} \mathrm{Ni}_{40} \mathrm{~B}_{20}$, Phys. Rev. Lett. 74 (1995) 2256.

[28] Z. Hu, Z.Q. Zhao, Y.D. Wu, et al., Surface features of Zr-based and Ti-based metallic glasses by ion irradiation, Vacuum 89 (2013) 142-146.

[29] R.C. Birtcher, S.E. Donnelly, S. Schlutig, Nanoparticle ejection from Au induced by single Xe ion impacts, Phys. Rev. Lett. 85 (2000) 4968-4971.

[30] S.E. Donnelly, R.C. Birtcher, Heavy ion cratering of gold, Phys. Rev. B 56 (1997) 13599-13602.

[31] K. Wien, Fast heavy ion induced desorption, Radiat. Effects Defects Solids 109 (1989) 137-167.

[32] I.A. Baranov, Y.V. Martynenko, S.O. Tsepelevich, et al., Inelastic sputtering of solids by ions, Soviet Phys. Uspekhi 31 (1988) 1015.

[33] E. Snoeks, T. Weber, A. Cacciato, et al., MeV ion irradiation - induced creation and relaxation of mechanical stress in silica, J. Appl. Phys. 78 (1995) 4723-4732.

[34] H. Trinkaus, Local stress relaxation in thermal spikes as a possible cause for creep and macroscopic stress relaxation of amorphous solids under irradiation, J. Nucl. Mater. 223 (1995) 196-201.

[35] P. Jung, Creep and electrical resistivity of metallic glass $\mathrm{Ni}_{78} \mathrm{~B}_{14} \mathrm{Si}_{8}$ under proton irradiation, J. Appl. Phys. 86 (1999) 4876-4880.

[36] P.G. Debenedetti, F.H. Stillinger, Supercooled liquids and the glass transition, Nature 410 (6825) (2001) 259-267.

[37] S.E. Orchard, On surface levelling in viscous liquids and gels, Appl. Sci. Res. $11 \mathrm{~A}(1962) 451$.

[38] F. Frost, R. Fechner, B. Ziberi, et al., Large area smoothing of surfaces by ion bombardment: fundamentals and applications, J. Phys.: Condens. Matter 21 (2009) 224026. 